Running head: EXTERNAL DEVICE AND GOAL-DIRECTED BEHAVIOUR

\title{
Is an external device efficacious to create and trigger intention in a patient with a severe brain injury?
}

Victorine Zermatten ${ }^{1,2,3}$, Lucien Rochat ${ }^{1,4}$, Rumen Manolov ${ }^{5,6}$ and Martial Van der Linden 1,4,

${ }^{1}$ Cognitive Psychopathology and Neuropsychology Unit, University of Geneva, Geneva, Switzerland

${ }^{2}$ Fondation Foyers Valais de Coeur, Sion, Switzerland

${ }^{3}$ Clinique Romande de Réadaptation, SUVA-Care, Sion, Switzerland

${ }^{4}$ Swiss Centre for Affective Sciences, University of Geneva, Geneva, Switzerland

${ }^{5}$ Department of Social Psychology and Quantitative Psychology, University of Barcelona, Barcelona, Spain

${ }^{6}$ Institute of Neurosciences, University of Barcelona, Barcelona, Spain

${ }^{7}$ Cognitive Psychopathology Unit, Psychology Department, University of Liège, Liège, Belgium

Word count: 6,788 (including references)

Lucien Rochat, PhD: lucien.rochat@unige.ch. Phone: 00413799346.

Rumen Manolov, PhD: rrumenov13@ub.edu. Phone: 0034934031137.

Martial Van der Linden, PhD: martial.vanderlinden@unige.ch. Phone: 0041223799341 
Running head: EXTERNAL DEVICE AND GOAL-DIRECTED BEHAVIOUR

Psychopathology and Neuropsychology Unit, Faculty of Psychology and Educational Sciences, University of Geneva, Boulevard du Pont d'Arve 40, CH-1205 Geneva, Switzerland. E-mail: Victorine.zermatten@unige.ch; Phone: 0041223799343 
Running head: EXTERNAL DEVICE AND GOAL-DIRECTED BEHAVIOUR

\section{Is an external device efficacious to create and trigger intention in a patient with a severe brain injury?}

Goal-directed behaviour (GDB), the capacity that allows us to control our environment according to our desires and needs, requires different stages from initial intention to goal achievement. Although GDB is frequently disrupted after acquired brain injury, few studies have addressed the remediation of reduced GDB in patients with severe brain injury. The present study aimed to raise this question in R.Z., a patient with severe brain injury who presented a serious reduction in GDB related to difficulties in creating an intention from internal determinants and in selecting an action plan, as well as to memory and attentional impairments. Our objective was to investigate the efficacy of an intervention programme to create and trigger a specific intention in R.Z. More specifically, this programme consisted in written prompts, first accompanied by an alarm provided by his mobile phone, but gradually reduced until only the alarm remained, which was implemented in order to improve R.Z.'s attendance at workshop sessions. Results showed that R.Z.'s attendance rate at the workshop sessions increased, indicating that the use of an external device allowed us to create and trigger intention in a patient with severe cognitive impairments.

Keywords: goal-directed behaviour, intention, rehabilitation, external device, single-case experimental design 
Running head: EXTERNAL DEVICE AND GOAL-DIRECTED BEHAVIOUR

\section{Introduction}

Goal-directed behaviour (GDB) refers to the capacity that allows us to control our environment according to our desires and needs (Dickinson \& Balleine, 1994) and is frequently disrupted after acquired brain injury (Lishman, 1978). The realisation of GDB requires different stages from the creation of an intention to goal achievement, including the action processes of selection, scheduling, and representation.

The GDB process starts with the creation of an intention either from internal determinants, such as a desire, wish, or urge, or from external determinants, such as a response to an environmental stimulus. In both cases, intentions have motivational properties that are either innate (positive reinforcement) or learned through experiences (previous success or failure). The probability of a behaviour occurring may be modified when these motivational properties emotionally label the behaviour. More specifically, previous success in the goal attainment and positive reinforcement associated with a particular behaviour produces a positive emotional response and increases the likelihood that this behaviour will be selected in the future. After the emergence of an intention, several processes are triggered in order to achieve different stages, including the selection of an appropriate serially ordered action programme, the initiation of this action plan, and the control of the outcome (for more details, see Brown, \& Pluck, 2000).

Thus, as GDB implies several mechanisms and consecutive phases, the reduction in GDB observed after an acquired brain injury may be the result of several disruptions during this process. Levy and Dubois (2006) proposed distinguishing between three forms of reduction in GDB, resulting from different underlying disrupted mechanisms: "auto-activation", "emotional-affective", or "cognitive". The first disruption is related to difficulties in the self-activation of thoughts, specifically to an 
Running head: EXTERNAL DEVICE AND GOAL-DIRECTED BEHAVIOUR

inability to reach the threshold of thought activation when an intention is created on an internal basis. Patients may describe a feeling of an empty mind and show total absence of spontaneous behaviour, but may exhibit the normal production of behaviours that are externally prompted (see, for example, patients with athymhormia described by Habib $\&$ Poncet, 1988). Consequently, these patients cannot create an intention from internal determinants, in contrast to the relatively preserved production of behaviour based on external determinants. Second, GDB reduction may also be explained by an inability to create a link between an emotion and the behaviour. Emotions are necessary to provide motivational values to the behaviour, which allow one to modify the probability of a behaviour occurring in the future. Thus, any change in the link between emotions and behaviours (e.g., if the patient exhibits emotional blunting) may produce a reduction in GDB. Finally, this deficit may also be related to impairments in several cognitive functions, especially executive (planning, set-shifting, rule finding, or inhibition), that are needed to elaborate a plan of action. Disruption during the initiation of this action plan also leads to GDB reduction because of an inability to translate this plan into action. In this case, the patient is able to describe a clear intention and to elaborate a plan of action, but not to translate it into an action.

Reduction in GDB is a frequent behavioural change observed after acquired brain injury, and a growing number of studies have focused on its revalidation in patients with mild to moderate cognitive deficits (see Boelen, Spikman, \& Fasotti, 2011 for a review). For instance, Goal Management Training (GMT; Robertson, 1996) aims at restoring the GDB process through the learning of a step-by-step procedure that helps patients to reformulate and achieve their goals. This training was proven to be efficient in the maintenance and realisation of intention in patients with mild to moderate cognitive deficits, especially when it was associated with other intervention strategies, 
such as external cueing, or when the GMT was implemented in daily life activities and not only in a laboratory task (Krasny-Pacini, Chevignard, \& Evans, 2014). For example, Tornås et al. (2016) showed that GMT associated with a daily text message that read "STOP" (the first stage of GMT), indicating to the participant that he had to interrupt his ongoing action to direct his attention towards a relevant goal, was effective in improving goal attainment in daily living activities in 33 patients who had chronic brain injury without severe cognitive impairment. Other studies showed the efficiency of an external device in the realisation of GDB, such as NeuroPage, a paging system that sends reminders according to prearranged schedules. More specifically, Evans, Emslie, and Wilson (1998) reported its efficiency in R.P., a 50-year-old woman who had ischaemic damage to the frontal lobes as a result of surgical clipping of two aneurysms. Despite the preservation of general intellectual and memory functioning, she presented difficulties in realising intended actions: She needed to be prompted to do things such as taking her medication or going to her voluntary job, even if she was able to say what she had to do. Her difficulties seemed to be related to difficulties during the translation of an action plan into action. NeuroPage was introduced to increase the probability of R.P. completing three tasks: taking medication, watering plants, and washing underwear. After 3 months of implementation, the pager was withdrawn for 3 weeks, and then reintroduced, to prove that positive effects were specifically related to the pager and not to other factors. Results showed a positive effect of the external device on the realisation of the three intended tasks and a reduction in performance when it was withdrawn. More than 10 years after this first study, Fish, Manly, and Wilson (2008) reported that R.P. was no longer using NeuroPage, which had been replaced after 5 years by an electronic self-programmed organiser. Although the organiser was initially successful in compensating for her day-to-day difficulties, its efficacy declined rapidly 
Running head: EXTERNAL DEVICE AND GOAL-DIRECTED BEHAVIOUR

and R.P.'s difficulties in daily living had returned without any change in her cognitive functioning. In particular, she spent a lot of time getting ready each morning and evening, and forgot to take her medication. Consequently, NeuroPage was reintroduced in order to compensate for these difficulties and was once more associated with significant functional gains.

In contrast to the growing number of interventions targeting GDB in patients with mild to moderate cognitive deficits, only a few studies have focused on patients with severe cognitive impairments. Two studies can be mentioned. Sohlberg, Sprunk, and Metzelaar (1988) investigated the efficacy of cue cards in a 38-year-old man who had sustained a severe traumatic brain injury (TBI) 13 months earlier, presenting executive impairments and being unable to resume his professional activity. He exhibited a reduction in GDB characterised by a restricted range of affect and little movement of any kind: He appeared to be apathetic and without motivation or drive, which seemed to indicate difficulties in the self-activation of thoughts. The intervention aimed at improving verbal and motor initiation during therapy group sessions, which took place during a day treatment programme for patients with acquired brain injury and consisted in prompting the patient through the use of a cue card. More specifically, during an initial individual training session, the patient was instructed in and learned to ask himself the question, “Am I initiating conversation?" when the therapist presented a card with a star on it, as well as the question, “Am I acknowledging other people's talking?" when the card had a circle on it. This strategy was then applied during nine therapy group sessions, and allowed him to increase the occurrence of both behaviours as shown by visual analysis. The study of Burke, Zencius, Wesolowski, and Doubleday (1991) confirmed and extended the conclusions of Sohlberg et al. (1988) in suggesting that the benefit related to the use of an external cueing procedure could be maintained 
even after its withdrawal. More specifically, Burke et al. (1991) aimed to improve selfgenerated behaviours by using a checklist in three patients with TBI. These patients presented severe executive impairments, especially deficits in problem-solving and planning, which impeded the elaboration of a plan of action and thus led to a reduction in GDB. In particular, they encountered difficulties in carrying out vocational or occupational activities requiring the achievement of several steps, without the assistance of a vocational specialist or a job coach. For one participant, the intervention was applied during his work at a local restaurant and for the other two, during the occupational activities occurring in a residential rehabilitation facility. Each participant received a checklist that stated in a sequential order the tasks and component to be completed. As shown by the results, which were based on visual analysis, the introduction of the checklist was proven to be efficient in reducing the number of verbal initiation cues provided by the job coach or the vocational specialist and thus to increase independence during work or occupational activities. When the checklist was withdrawn, the number of verbal initiation cues remained at a stable level, suggesting that with practice, the checklist contributed to the installation of a lasting action routine.

Except for these two interventions, to our knowledge, no other research has addressed the question of GDB revalidation in patients with severe cognitive impairments. Considering the need for further studies, we describe an intervention programme that aimed to improve the realisation of GDB in R.Z., a patient with severe brain injury living in a sheltered home and presenting severe cognitive impairments. R.Z. exhibited a serious GDB reduction that appeared to result from difficulties during both the creation of an intention based on internal determinants and from the selection and scheduling of an action plan, along with episodic memory and attentional impairments. The objective of the current study was to create and trigger a specific 
Running head: EXTERNAL DEVICE AND GOAL-DIRECTED BEHAVIOUR

action intention in R.Z. More specifically, the intervention programme consisted in written prompts, first accompanied by an alarm provided by his mobile phone, but gradually reduced until only the alarm remained, which was implemented in order to improve R.Z.'s attendance at workshop sessions. The efficacy of the intervention was assessed by means of both visual analysis and quasi-statistical analysis.

\section{Case history}

R.Z. is a 42-year-old, right-handed man. Born in France, he lived for the past 25 years in the French-speaking part of Switzerland. He completed compulsory school in France and interrupted high school before his graduation year. Since his arrival in Switzerland, he worked in a paint factory and then as a lifeguard.

In April 2012, he had a subarachnoid haemorrhage caused by the rupture of an aneurysm on the anterior communicating artery, treated by clipping. An initial slow recovery was aggravated by cerebral oedema that required a craniotomy and installation of an external right ventricular drain. Other complications arose thereafter, including cerebral arterial vasospasm, brain swelling, epilepsy, surgical site infections upon delivery of the bone flap, broncho-aspiration and pneumonia, central diabetes insipidus, and hydrocephalus. Cerebral arterial vasospasms caused ischaemic lesions in the territory of the anterior cerebral artery (bilaterally), as well as within the right temporopolar region. External ventricular drains had to be changed several times. The last computed tomography scan performed before the intervention (in October 2012) confirmed the correct position of the external ventricular drain and the persistence of ischaemic lesions. Because of persistent motor difficulties (right-side hemiparesis) and severe cognitive and behavioural impairments, including a severe reduction of GDB, R.Z. was admitted to a sheltered home in 2013. Since his admission, he had attended the occupational workshop, which took place in a building adjacent to the home. A personal 
workshop schedule is developed for each nursing home resident, depending on their cognitive functioning, wishes, and fatigability. Various activities are proposed at the workshop, including horticulture, crafting wood, or cooking. In order for the residents to know which activity to go to, each of their workshop schedules is displayed in each room or on the information board next to the workshop location.

Since he began residing in the sheltered home, reports from the staff indicated that R.Z. showed a lack of self-initiated actions and that he needed constant prompting to, for example, take his medication, move around independently in the sheltered home, transfer from his wheelchair to his bed, clean his teeth, or go alone to workshop sessions. If prompted, R.Z. had the ability to perform these simple actions and he knew exactly where to go to take his medication or how to do the transfers from his wheelchair to his bed. His spontaneous speech was also extremely reduced: He rarely initiated conversation and his responses to questions were sparse, consisting either of single words or single sentences. During the workshop sessions, R.Z. needed great prompting to begin a task, whereas he was able to independently maintain it until its achievement. However, when he finished a task, he remained inactive without informing the staff that it had been achieved, which impeded him from pursuing another task.

The absence of self-initiated behaviour was associated with a number of adverse outcomes. In particular, the need for external stimulation in order to perform tasks that he had the ability to carry out prevented him from developing autonomy in the sheltered home, including going alone to the workshop sessions. More specifically, R.Z. needed to be reminded at the appropriate moment of every workshop day to go to the sessions; when he was not prompted by a staff member, he remained in his room. Considering that the staff did not always have the time to prompt him, he did not go to every 
Running head: EXTERNAL DEVICE AND GOAL-DIRECTED BEHAVIOUR

workshop session. However, R.Z. knew where the workshop took place and that he had to go, without being able to say exactly when.

\section{Neuropsychological assessment}

A neuropsychological assessment performed 6 months after his admission to the home showed severe impairments in executive functions (planning, set-shifting, problem solving, inhibition), severe deficits in verbal episodic memory, slowing of reaction times and attentional impairments (divided and sustained attention), and mild left hemineglect (see Table 1). Spatio-temporal orientation, oral expression and language comprehension, and verbal and visual short-term memory were globally preserved. Finally, R.Z. displayed no constructive or ideomotor praxic disorders.

\section{--INSERT TABLE 1 HERE--}

\section{Interpretation of R.Z.'s reduction in GDB}

R.Z.'s reduction in GDB could be explained as an association of impairments. In particular, daily living observations showed that R.Z. did not spontaneously initiate an intention, despite the fact that when the staff indicated to him the action to be performed, he was able to carry it out. In other words, R.Z. had the ability to perform everyday actions whenever he was instructed to do so, such as to take his medication, to make transfers from his wheelchair to his bed, or to go alone to the workshop sessions. Furthermore, he was motivated to achieve all these tasks and knew how to carry them out. In particular, he was pleased to work during the workshop sessions and he had even accepted an increase in his workshop schedule. The combination of all these observations suggested that R.Z.'s reduction in GDB may be first explained by an inability to create an intention from internal determinants, in contrast to the normal 
production of behaviours that are externally prompted. Furthermore,

neuropsychological assessment, especially the identification of planning impairment, suggested that R.Z.'s reduction in GDB may also be related to a disruption in the selection and scheduling of an action plan. Finally, episodic memory and attentional impairments could also contribute to the reduction in GDB through an inability to remember the goal to achieve, distracted behaviour, and a drift from the intended goal.

\section{Rationale for the intervention}

An intervention programme composed of three steps that specifically target or bypass these impairments was implemented in order to create and trigger a specific intention in R.Z. More specifically, the aim of the intervention was to improve the number of times that he attended the sheltered workshop per week (in terms of percentage) without the help of someone else. During the first step of the intervention programme, the description of the to-be-performed action, accompanied by an auditory alarm, was provided to him through the use of his mobile phone at the specific moment when the action had to be carried out. The written prompt indicated the activities where R.Z. was awaited. This strategy was used in order to compensate R.Z.'s memory and attentional impairments: Written prompts acted as a reminder for the action of going to the workshop sessions, and the auditory alert attracted R.Z.'s attention to the to-beperformed intention. During the second stage of the intervention, the message was shortened to simply indicate the activities where he was awaited. This approach of gradually diminishing cues was used to progressively create an internal association between the alarm and the to-be-performed intention. Finally, during the last step of the intervention, the message was removed and only the alarm remained. We hypothesised that after the auditory alert was associated with the action of going to the workshop sessions, the simple alarm could trigger the intention and the realisation of this action. 
Running head: EXTERNAL DEVICE AND GOAL-DIRECTED BEHAVIOUR

However, considering R.Z.'s memory impairments, even if he were able to learn the meaning of the alarm (indicating that he had to go the workshop sessions), he would encounter difficulties in remembering the activity where he was awaited. Consequently, it was decided that when he asked the staff which activity he had to go to, he would be encouraged to check the workshop schedule displayed in his room or on the information board next to the workshop location.

\section{Intervention design}

Initially, one $\mathrm{ABAB}$ single-case experimental design was planned. However, during the intervention, the number of R.Z.'s workshop sessions increased and the intervention programme was modified accordingly, which led to the implementation of a second $\mathrm{ABAB}$ single-case experimental design. Each phase included a minimum of three measurement time points, as recommended by Beeson and Robey (2006). Outcome measures were completed every day of the workshop session.

\section{--INSERT FIGURE 1 HERE-}

\section{First intervention programme}

During the first part of the intervention, R.Z.'s weekly workshop schedule included three sessions in two different activities: He attended the workshop on Mondays, Thursdays, and Fridays (see Figure 1). During 4 weeks of baseline (baseline; 12 workshop sessions), R.Z. had his mobile phone with him, but no reminder was programmed, and he was encouraged to carry his phone with him to create a habit of taking it before going to the workshop sessions. The first intervention programme was then implemented. During the first step of the first intervention programme (B1 part 1), which lasted 6 weeks (18 workshop sessions), his phone was programmed to bleep 
Running head: EXTERNAL DEVICE AND GOAL-DIRECTED BEHAVIOUR

loudly and to display a reminder message indicating where he had to go (e.g., "R.Z., you are awaited at the horticulture workshop"). R.Z. had received the instruction to read the message and to follow the written instructions. The content of the messages changed according to the workshop session activity. The second step of the intervention (B1 part 2) was then introduced and lasted 4 weeks (12 workshop sessions interspersed by 2 weeks of holiday). During this step, the message was shortened to simply indicate the activities where he was awaited (e.g., "Horticulture workshop"). The alarm and the instruction remained the same. Finally, the last step (B1 part 3) lasted 6 weeks (18 workshop sessions). During this step, the messages were removed and only the alarm remained. R.Z. was told that he would receive no more messages, but that he still had to go to the correct workshop sessions when the alarm rang. The alarm was then withdrawn for 3 weeks (nine workshop sessions) (A2). After its removal, the alarm was reintroduced for 25 weeks for the sessions occurring on Thursdays and Fridays (B2) (50 workshop sessions).

Because of R.Z.'s motivation to participate in the workshop sessions, the staff decided to increase the number of his sessions, which required an adaptation of the study design and the implementation of a second intervention programme.

\section{Second intervention programme}

During the second part of the intervention, R.Z.'s weekly workshop schedule included two supplementary sessions in two different activities, and the activity taking place on Mondays was modified. Consequently, during the second intervention programme, R.Z. attended the workshop on Monday and Tuesday mornings and afternoons (see Figure 1). The intervention procedure was the same as the first intervention programme: After a 3-week period of baseline (second baseline) (nine workshop sessions), the intervention was implemented: The first step of the intervention (messages and alarm; 
Running head: EXTERNAL DEVICE AND GOAL-DIRECTED BEHAVIOUR

second B1 part 1) lasted 4 weeks (12 workshop sessions), the second step (shortened messages and alarm; second B1 part 2) 5 weeks (15 workshop sessions), and the last step (alarm; second B1 part 3) 15 weeks (45 workshop sessions interspersed by two holiday periods and one hospitalisation period). The alarm was then withdrawn for 5 weeks (second A2) (15 workshop sessions). After its removal, the alarm was reintroduced for 5 weeks (second B2) (15 workshop sessions).

\section{Measures}

Two types of measures were used to assess the efficacy of the intervention. The primary outcome measure involved the target behaviour. For every workshop sessions, the persons in charge of the workshop activities recorded the presence or absence of R.Z. in a table. If R.Z. was not present 15 min after the start time, he was considered to be absent. After this limit, staff members were instructed to prompt R.Z. to go to the workshop. Given that the intervention was performed in a clinical setting, some of R.Z.'s absences were related to medical appointments, hospitalisations, or holidays and were not due to his lack of self-initiated action or his memory impairments. These absences were not included in the data processed for statistical purposes.

The secondary outcome measures involved questionnaires and qualitative observations. First, in order to assess a change in his executive functioning, specifically in the initiation deficit, the Dysexecutive Questionnaire (DEX; Wilson, Alderman, Burgess, Emslie, \& Evans, 1996) was completed by R.Z., his mother, and his nurse before the intervention and 3 months after the intervention. The DEX is a 20-item questionnaire that assesses behavioural issues following acquired brain injury associated with a dysexecutive syndrome. We used the clinical severity classification proposed by Bodenburg and Dopslaff (2008), who identified four factors of the DEX: "initiating and sustaining actions" (e.g., "I will say one thing, but do something different"), "impulse 
Running head: EXTERNAL DEVICE AND GOAL-DIRECTED BEHAVIOUR

control and sequencing of action" (e.g., "I get events mixed up with each other, and get confused about the correct order of events"), "excitability" (e.g., "I tend to be very restless, and can't sit still for any length of time"), and "social convention and the ability to incorporate social interaction in own behaviour" (e.g., "I do or say embarrassing things when in the company of others"). This classification allowed us to characterise the severity of the dysexecutive syndrome at one of three levels; it was considered severe if the total score was between 29 and 36, moderate if the score was between 21 and 28, and mild if the score was between 0 and 20 .

Second, information concerning R.Z.'s daily functioning was obtained through informal interviews conducted with the staff. Once per month, the referral team of each patient (including one nurse, one social educator, and one person in charge of the workshop) met in order to assess progress in the patient's daily functioning and to identify the functional tasks that he or she could carry out more independently. Qualitative observations of R.Z.'s functioning after the intervention were used as clinical indicators of the intervention effects.

\section{Analyses}

To examine the efficacy of the intervention, we first performed visual analyses involving the examination of level, trend, and variability of R.Z.'s attendance at the workshop sessions (Kratochwill et al., 2010); these analyses were crucial to gain a first general impression of the data. Structured visual analysis involves assessment of withinphase trend, level, and stability of data, as well as a comparison of the same data features across conditions and an assessment of the overlap between the data and the immediacy of the effect of changing the conditions. The changes observed between two conditions (e.g., baseline to intervention, intervention to baseline) in the trend, level, or 
Running head: EXTERNAL DEVICE AND GOAL-DIRECTED BEHAVIOUR

stability of the data indicate whether the introduction of the intervention is related to the change in behaviour occurrence.

We complemented these visual analyses by using a specific quasi-statistical technique (a combination deemed to be appropriate by Tate et al., 2013) called Tau-U, which measures the non-overlap between two phases and quantifies the improvement in terms of percentage (Parker, Vannest, Davis, \& Sauber, 2011), while taking into account and controlling for the possible trend in the baseline. According to Parker, Vannest, and Davis (2011), Tau-U is the most statistically powerful index of those available. Thus, Tau-U allowed us to quantify the amount of improvement in the intervention phase and to investigate whether there was a significant increase (in percentage) of R.Z.'s attendance at the workshop. For computing Tau-U, we used the R code provided in Brossart, Vannest, Davis, and Patience (2014). In a withdrawal design such as in the current study, the Tau-U effect size can be computed for each baseline and intervention pair.

\section{Results}

Two data sets were analysed, corresponding to the two A-B-A-B designs (first and second intervention programmes). The first B phase included data from the reminders and alarm (B1 part1), from the reduction of the message (B1 part 2), and from the alarm only (B1 part 3). We chose to gather data from the three parts of the intervention during the first B phase because there was no difference in level between them. The second B phase contained data only from the alarm. Data sets entailed one data point per week, presented as a percentage (e.g., 100\% means that R.Z. correctly attended all sessions).

First intervention programme (First $A B A B$ design)

Structured visual analysis 
Figures 2 and 3 represent R.Z.'s presence at the workshop in terms of percentage, obtained by using the SCDA plug-in (Bulté \& Onghena, 2012) for R (R Core Team, 2013) and later modified by adding the dashed line representing median and trend (ordinary least squares). Regarding baseline stability, the initial baseline already showed an upward trend. The introduction of the intervention (B1) was associated with a slope that was not as steep as the baseline slope. It was only with the device, in phase B1, that $100 \%$ attendance was achieved and thus no further improvement (and no steeper slope) was possible in the intervention phase. In addition, after the 2-week holiday period of the workshop, no decrease in R.Z.'s attendance was observed in the data (after the eighth data point of the B1 phase). Withdrawal of the intervention (A2) was associated with an immediate decrease in attendance. Nevertheless, a clearer upward trend shows that attendance progressively increased after the withdrawal (Figures 2 and 3). Finally, the second intervention phase (B2) shows, even more clearly than the first $A B$ comparison does, that $100 \%$ attendance was achieved and sustained only during the intervention, with a single outlying value being different from the maximal possible attendance. Again, no decrease in attendance was observed after R.Z.'s two holiday periods and after his hospitalisation for 3 weeks.

In general, the introduction of the intervention (B1) was associated with a change in the level of R.Z.'s presence at the workshops (an increase in percentage). As shown in Figure 3, the median level increased from $16.5 \%$ in $A_{1}$ to $100 \%$ in $B_{2}$ and from $66 \%$ in $\mathrm{A}_{2}$ to $100 \%$ in $\mathrm{B}_{2}$.

Visually, the amount of overlap was small; quantitative analyses are presented in the following section. Finally, regarding immediacy, 100\% attendance was achieved in the first or second week of intervention, while the drop in attendance was also immediate when the intervention was withdrawn. 
Running head: EXTERNAL DEVICE AND GOAL-DIRECTED BEHAVIOUR

--INSERT FIGURES 2 \& 3 HERE--

\section{Quasi-statistics}

The quantifications of aspects of the visual analysis, in which we used percentage of data overlap, are presented here. Three Tau-U scores were computed because of the $\mathrm{AB}^{2}$ design: $\mathrm{A} 1-\mathrm{B} 1, \mathrm{~B} 1-\mathrm{A} 2$, and $\mathrm{A} 2-\mathrm{B} 2$. The results indicate that with the introduction of the intervention, Tau-U was .85 (i.e., the percentage of data non-overlap between the first baseline and the first B phase, after the monotonic phase A trend was accounted for, was $85 \%)$, a statistically significant result $(p<.01)$. With the withdrawal of the alarm, Tau-U was -.20 , not reaching statistical significance $(p=.07)$. With the reintroduction of the alarm (B2), Tau-U was .52, a statistically significant result $(p<$ .01). All Tau values were, therefore, in the expected direction. Taken together, the results of Tau-U indicate that the first phase of the intervention (B1) was more effective than the second (B2), as .85 can be interpreted as a very large change and .52 as a moderate one (Vannest \& Ninci, 2015). Moreover, the percentage of weeks with $100 \%$ attendance was $95.65 \%$ in B2, whereas the percentage of weeks with $100 \%$ attendance was $86.67 \%$ in B1. Therefore, taking all these results into account, it can be concluded that during the first intervention programme, the second intervention phase (B2) consolidated the results of the first phase (B1), both being different from baseline or withdrawal levels of attendance.

\section{Second intervention programme (Second ABAB design)}

The same analyses were performed with the second data set.

Structured visual analysis

Figures 4 and 5 represent the percentage of attendance at the workshop. Again, the ordinary least squares fitted line suggests an improving trend, but there are still no 
consecutive improvements in workshop attendance. The introduction of the intervention (B1) was associated with a change in level, that is, an increase in the percentage of R.Z.'s presence at the workshops from a median of $33 \%$ to a median of $100 \%$. As in the first intervention programme, the maximum was reached repeatedly during the intervention (B1), yet at a reduced frequency in comparison to the first intervention programme. After R.Z.'s two holiday periods and his hospitalisation, no significant decrease in attendance was observed in the data (after the 13th, 16th, and 20th data point of the B1 phase). Withdrawal of the intervention (A2) was associated with a decrease in attendance, according to the downward trend. Again, with the reintroduction of the intervention (B2), R.Z.'s presence at the workshops increased, as shown by the upward trend. More important, the percentage of weeks with $100 \%$ attendance increased from $60 \%$ to $80 \%$, including the last 4 weeks of the study. No decrease was observed after the holiday period of the workshop (third data point of the B2 phase). Finally, the effects of the intervention were not as immediate as during the first intervention programme.

\section{--INSERT FIGURES 4 \& 5 HERE--}

\section{Quasi statistics}

Three Tau-U scores were computed because of the $\mathrm{AB}^{2}$ design: A1-B1, B1-A2, and A2-B2. The results indicate that with the introduction of the intervention, Tau-U was .72 (i.e., the percentage of data non-overlap between the first baseline and the first B phase, after the monotonic phase A trend was accounted for, was 72\%), a statistically significant effect $(p<.05)$. With the withdrawal of the alarm, Tau-U was $.00(p=.98)$. With the reintroduction of the alarm (B2), Tau-U was .11, a non-significant effect ( $p=$ .78). 
Running head: EXTERNAL DEVICE AND GOAL-DIRECTED BEHAVIOUR

As was the case for the first $\mathrm{AB}^{2}$, the Tau results for the second $\mathrm{AB}^{2}$ design indicate that the first introduction of the warning signal (a large change according to the interpretative criteria suggested by Vannest and Ninci, 2015) was more effective than the second (a less than small change), even though the percentage of weeks with $100 \%$ attendance was $56.52 \%$ in B1 and $80 \%$ in B2. Moreover, the non-overlap was greater for the first intervention programme than for the second.

\section{Questionnaire and qualitative outcomes}

The DEX scores are presented in Table 2. The score on the "initiate, sustain" factor decreased after the intervention, especially according to the patient's assessment. This decrease was more important than for the other factors. However, despite this reduction, the score remained at a high level, indicating the persistence of a severe initiation deficit.

--INSERT TABLE 2 HERE--

During an informal interview conducted after the intervention, the persons in charge of the workshop and the nurses confirmed that the intervention was effective in increasing R.Z.'s attendance at the workshops. After the intervention, he no longer needed to be reminded on each workshop day, at the appropriate moment, to go to the workshop session. Even without the alarm, his attendance rate at the workshop sessions remained high. During these sessions, when he had finished a task, he spontaneously contacted the staff in order to pursue another task. Additionally, other aspects of GDB, such as independently moving around in the sheltered home and taking his medication, also improved, as well as his spontaneous speech. He could initiate brief conversations with other patients. However, outside the context of the sheltered home and without the 
Running head: EXTERNAL DEVICE AND GOAL-DIRECTED BEHAVIOUR

use of his mobile phone, constant prompting was always needed to manage his appointments and to implement intentions.

\section{Discussion}

The aim of the present study was to investigate the efficacy of an external cueing system to create and trigger intention in R.Z., a 42-year-old patient presenting reduction in GDB and severe cognitive impairments and living in a sheltered home. We hypothesised that R.Z.'s reduction in GDB resulted from perturbations during both the creation of an intention from internal determinants and the selection and scheduling of an action plan, along with episodic memory and attentional impairments. The intervention programme was implemented in order to increase R.Z.'s attendance at the workshop sessions and was designed in three steps: the delivery of an alarm and the description of the to-be-performed action, the reduction in the reminders, and finally, the alarm only. These three steps were intended to bypass R.Z.'s memory and attentional impairments, to create an association between the alarm and the to-beperformed intention, and finally to trigger the intention and the action of going to the workshop sessions.

The results showed that the intervention led to a significant increase in R.Z.'s attendance rate at the workshop sessions, indicating that the intervention programme was efficient in creating and triggering an intention to action in this patient. Both visual and quasi-statistical analyses suggested an improvement in the attendance rate at the workshop sessions over baseline after the alarm and the message were introduced. This result was supported by informal interviews conducted with the staff after the intervention and to a lesser extent by the slight decrease observed in the "initiate and sustain" factor of the DEX. 
Running head: EXTERNAL DEVICE AND GOAL-DIRECTED BEHAVIOUR

The maintenance of the significant improvement in R.Z.'s attendance at the workshop sessions that was observed when only the alarm remained for the first intervention programme may be explained by the fact that the action of going to the workshop sessions became a conditioned response. More specifically, during the first two steps of the intervention (reminders and alarm; reduction of the message and alarm), the action of going to the workshop sessions was progressively associated with the auditory alert. After this link was established, the simple alarm automatically triggered the intention of the to-be-performed action. The auditory alert probably also provoked an interruption in R.Z.'s ongoing action, allowing him to draw his attention to the meaning of the alarm, which triggers the intention and the targeted action. This interpretation is in accordance with the work of Manly, Hawkins, Evans, Woldt, and Robertson (2002). They showed that the provision of an unexpected tone without any specific content about the activity to be carried out allowed improvement in the performance of the Hotel task in 10 patients with mild to severe brain injury. During this task, the participants had to carry out six distinct activities related to running a hotel (e.g., compiling individual bills or looking up telephone numbers) in $15 \mathrm{~min}$. The improvement in the patients' performance in this task was interpreted to result from the fact that the unexpected tone would interrupt the task at hand, allowing the patient to think about the goal to be achieved and to redirect his attention towards it, which consequently influenced his subsequent behaviour and allowed better performances during a task. In our study, the auditory alert probably served a similar purpose, interrupting R.Z.'s current action and drawing his attention to think about the specific intention to be performed.

It should be noted that R.Z.'s attendance rate was lower during the second than it was during the first intervention programme. This result may be related to his memory 
impairments. When the second intervention programme was introduced following an increase in R.Z.'s number of workshop sessions, modification of his schedule required him to store and remember this new information. During the first two steps of the intervention (reminders and alarm; reduction of the message and alarm), R.Z. knew exactly the activity where he was awaited. However, when only the alarm remained for the first time during this second programme (B1 part 3), variations in his attendance rate were observed. The auditory alert probably triggered the intention to go to the workshop sessions, but, given his memory impairments, R.Z. encountered difficulties in precisely identifying the activity in which he was awaited. Consequently, he did not go to every workshop session during the first introduction of the intervention for the second intervention programme. Provision of the written prompt for more than 9 weeks may have been needed to ensure adequate learning of his new workshop schedule.

Why R.Z.'s attendance rate at the workshop sessions was more stable during the last phase of the second intervention programme (B2 phase) remains to be examined. During this phase, only the alarm was provided to R.Z. and at that moment, members of the staff reported that R.Z. began to solicit them in order to know where he had to go when he heard the alarm. Because he knew the meaning of the alarm, but not the activity where he was awaited, he asked the staff members, who were instructed to send him back to his workshop schedule displayed in his room or on the information board next to the workshop location. Progressively, R.Z. used this strategy spontaneously every time he heard the alarm, without asking the staff. Consequently, an internal association between the alarm, the intention of going to the workshop sessions, and the checking strategy was probably created, which may explain his stable attendance rate during the last phase of the intervention for the second intervention programme. 
Running head: EXTERNAL DEVICE AND GOAL-DIRECTED BEHAVIOUR

It is also interesting to note that along with an increase in R.Z.'s attendance rate at the workshop sessions, an improvement in the realisation of other GDBs was reported by the staff. More specifically, R.Z. showed an improvement in the action of moving independently around in the sheltered home and of taking his medication. These secondary benefits of the intervention may be explained by the fact that the increase in R.Z.'s attendance rate at the workshop sessions probably impacted on his self-esteem. The increase in his self-esteem was reflected in the fact that he reported his attendance at the workshop sessions and appeared to be proud of this. Because R.Z. was praised by the persons in charge of the workshop session when he attended the correct activity, positive emotions were probably produced. Success in goal achievement (i.e., to attend the correct activity at the workshop) and positive reinforcement provided by the staff could have increased his self-esteem, which then probably impacted on motivation and on the realisation of other GDBs.

Finally, the limitations of this study also need to be acknowledged, especially regarding the analysis of the data. Because the baseline contained only three data points showing variability during the two intervention programmes (baseline and withdrawal of the alarm), this may have affected the comparison between the levels (medians) of the different conditions. The use of a longer baseline might have helped to resolve this issue. However, it has been difficult to increase the duration of the baseline considering the clinical setting in which the intervention was applied: Attendance at the workshop sessions was an integral part of R.Z.'s daily functioning and it was important to him to follow his workshop sessions. Moreover, although we chose a non-overlap index for quantification according to the features of the data at hand (Manolov \& Moeyaert, 2016), there is still no consensus about the optimal analytical techniques (Kratochwill et al., 2010; Tate et al., 2013), which could open any choice to criticism. 
Running head: EXTERNAL DEVICE AND GOAL-DIRECTED BEHAVIOUR

Despite these limitations, the intervention programme described in this study was efficient to create and trigger intention in a patient with severe cognitive impairments and a severe reduction in GDB. This programme consisting of the delivery of written prompts, first accompanied by an alarm provided by his mobile phone but gradually reduced until only the alarm remained, allowed us to increase the patient's attendance rate at the workshop sessions and thus improve his autonomy in the sheltered home. In addition to the creation of an action intention in the patient, the programme also led to the implementation of a checking strategy that consisted in consulting the workshop schedule displayed in R.Z.'s room. The use of this strategy allowed him to maintain the benefit of the intervention when only the alarm remained. 
Running head: EXTERNAL DEVICE AND GOAL-DIRECTED BEHAVIOUR

\section{Acknowledgments}

The authors declare no conflict of interest. 
Running head: EXTERNAL DEVICE AND GOAL-DIRECTED BEHAVIOUR

\section{References}

Beeson, P., \& Robey, R. (2006). Evaluating single-subject treatment research: Lessons learned from the aphasia literature. Neuropsychological Review, 16, 139-161.

Bodenburg, S., \& Dopslaff, N. (2008). The Dysexecutive Questionnaire advanced: Item and test score characteristics, 4-factor solution, and severity classification. The Journal of Nervous and Mental Disease, 196, 75-78.

Boelen, D. H. E., Spikman, J. M., \& Fasotti, L. (2011). Rehabilitation of executive disorders after brain injury: Are interventions effective? Journal of Neuropsychology, 5, 73-113.

Brossart, D. F., Vannest, K., Davis, J., \& Patience, M. (2014). Incorporating nonoverlap indices with visual analysis for quantifying intervention effectiveness in single-case experimental designs. Neuropsychological Rehabilitation, 24, 464-491.

Brown, R. G., \& Pluck, G. (2000). Negative symptoms: The 'pathology' of motivation and goal-directed behaviour. Trends in Neurosciences, 23, 412-417.

Bulté, I., \& Onghena, P. (2012). When the truth hits you between the eyes: A software tool for the visual analysis of single-case experimental data. Methodology, 8, 104114.

Burgess, P. W., \& Shallice, T. (1996). Bizarre responses, rule detection and frontal lobe lesions. Cortex, 32, 241-259.

Burke, W. H., Zencius, A. H., Wesolowski, M. D., \& Doubleday, F. (1991). Improving executive function disorders in brain-injured clients. Brain Injury, 5, 241-252.

D’Elia, L. F., Satz, P., Uchiyama, C. L., \& White, T. (1996). Color Trails Test professional manual. Odessa, FL: Psychological Assessment Resources. 
Running head: EXTERNAL DEVICE AND GOAL-DIRECTED BEHAVIOUR

Dickinson, A., \& Balleine, B. (1994). Motivational control of goal-directed action. Animal Learning \& Behavior, 22, 1-18.

Evans, J. J., Emslie, H., \& Wilson, B. A. (1998). External cueing systems in the rehabilitation of executive impairments of action. Journal of the International Neuropsychological Society, 4, 399-408.

Fish, J., Manly, T., Emslie, H., Evans, J. J., \& Wilson, B. A. (2008). Compensatory strategies for acquired disorders of memory and planning: Differential effects of a paging system for patients with brain injury of traumatic versus cerebrovascular aetiology. Journal of Neurology, Neurosurgery \& Psychiatry, 79, 930-935.

Fish, J. E., Manly, T., \& Wilson, B. A. (2008). Long-term compensatory treatments of organizational deficits in a patient with bilateral frontal lobe damage. Journal of the International Neuropsychological Society, 14, 154-163.

Gauthier, L., Dehaut, F., \& Joanette, Y. (1989). The Bells Test: A quantitative and qualitative test for visual neglect. The International Journal of Clinical Neuropsychology, 11, 49-54.

GREFEX: Groupe de Réflexion sur 1'Evaluation des Fonctions Exécutives (Azouvi, P., Didic-Hamel, C. M., Fluchaire, I., Godefroy, O., Hoclet, E., Le Gall, D., ...Pillon, B.). (2001). L'évaluation des fonctions exécutives en pratique clinique. Revue de Neuropsychologie, 11, 383-433.

Habib, M., \& Poncet, M. (1988). Perte de l'élan vital, de l'intérêt et de l'affectivité (syndrome athymhormique) au cours de lésions lacunaires des corps striés. Revue neurologique, 144, 571-577.

Hooper, H. E. (1958). The Hooper visual organization test: Manual. Beverly Hills, CA: Western Psychological Services. 
Running head: EXTERNAL DEVICE AND GOAL-DIRECTED BEHAVIOUR

Kratochwill, T. R., Hitchcock, J., Horner, R. H., Levin, J. R., Odom, S. L., Rindskopf, D. M., \& Shadish, W. R. (2010). Single-case design technical documentation. Retrieved from What Works Clearinghouse website: http://ies.ed.gov/ncee/wwc/pdf/wwc_scd.pdf

Krasny-Pacini, A., Chevignard, M., \& Evans, J. (2014). Goal Management Training for rehabilitation of executive functions: a systematic review of effectivness in patients with acquired brain injury. Disability and rehabilitation, 36, 105-116.

Levy, R., \& Dubois, B. (2006). Apathy and the functional anatomy of the prefrontal cortex-basal ganglia circuits. Cerebral Cortex, 16, 916-928.

Lishman, W. A. (1978). Organic psychiatry: The psychological consequences of cerebral disorders. Boston, MA: Blackwell Scientific.

Manolov, R., \& Moeyaert, M. (2016, May 16). Recommendations for choosing singlecase data analytical techniques. Behavior Therapy. Advance online publication.

Parker, R. I., Vannest, K. J., \& Davis, J. L. (2011). Effect size in single-case research: A review of nine nonoverlap techniques. Behavior Modification, 35, 303-322.

Parker, R. I., Vannest, K. J., Davis, J. L., \& Sauber, S. B. (2011). Combining onoverlap and trend for single-case research: Tau-U. Behavior Therapy, 42, 284-299.

Poitrenaud, J., Deweer, B., Kalafat, M., \& Van der Linden, M. (2007). Adaptation en langue française du California Verbal Learning Test [French adaptation of the California Verbal Learning Test]. Paris, France: Les Éditions du Centre de psychologie appliquée.

R Core Team. (2013). R: A language and environment for statistical computing. Vienna, Austria: R Foundation for Statistical Computing. Retrieved from http://www.R-project.org/ 
Running head: EXTERNAL DEVICE AND GOAL-DIRECTED BEHAVIOUR

Regard, M., Strauss, E., \& Knapp, P. (1982). Childen's production on verbal and nonverbal fluency tasks. Perceptual and Motor Skills, 55, 839-844.

Robertson, I. H. (1996). Goal Management Training: A clinical manual. Cambridge, U.K.: PsyConsult.

Shallice, T. (1982). Specific impairments of planning. Philosophical Transactions of the Royal Society of London: Biological Sciences, 298, 199-209.

Sohlberg, M. M., Sprunk, H., \& Metzelaar, K. (1988). Efficacy of an external cuing system in an individual with severe frontal lobe damage. Cognitive Rehabilitation, 6, $36-41$.

Tate, R. L., Perdices, M., Rosenkoetter, U., Wakima, D., Godbee, K., Togher, L., \& McDonald, S. (2013). Revision of a method quality rating scale for single-case experimental designs and n-of-1 trials: The 15-item Risk of Bias in N-of-1 Trials (RoBiNT) Scale. Neuropsychological Rehabilitation, 23, 619-638.

Thuillard, F., \& Assal, G. (1989). ENEC (examen neuropsychologique du CHUV) chez la personne vieillissante [Neuropsychological assessment in the aging person]. Bulletin d'audiophonologie, 5, 373-381.

Tornås, S., Løvstad, M., Solbakk, A.-K., Evans, J., Endestad, T., Hol, P. K., ...Stubberud, J. (2016). Rehabilitation of executive functions in patients with chronic acquired brain injury with Goal Management Training, external cuing, and emotional regulation. Journal of the International Neuropsychological Society, 22, 436-452.

Vannest, K. J., \& Ninci, J. (2015). Evaluating intervention effects in single-case research designs. Journal of Counseling \& Development, 93, 403-411.

Wilson, B.A., Alderman, N., Burgess, P.W., Emslie, H., \& Evans, J.J. (1996). Behavioural assessment of the dysexecutive syndrome. Bury St. Edmunds, United Kingdom: Thames Valley Test. 
Running head: EXTERNAL DEVICE AND GOAL-DIRECTED BEHAVIOUR

Zimmermann, P., \& Fimm, B. (2009). Testbatterie zur Aufmerksamkeitsprüfung (TAP)

[Tests battery for attention assessment] (Version 2.2). Herzogenrath, Germany: Psytest. 
Running head: EXTERNAL DEVICE AND GOAL-DIRECTED BEHAVIOUR

Table 1. Impaired performances of R.Z.

\begin{tabular}{llll}
\hline Test & Raw score & $\begin{array}{l}\text { Normative } \\
\text { data mean } \\
(S D)^{*}\end{array}$ & $\begin{array}{l}\text { Performance } \\
\text { descriptors** }^{*}\end{array}$ \\
\hline Visuospatial perception & $12.5 / 20$ & 14.5 & Impaired \\
$\begin{array}{c}\text { Hooper Visual Organization Test }{ }^{1} \\
\begin{array}{c}\text { Bells Test } \\
\text { : } \text { omissions (total }\end{array}\end{array}$ & 4 & 6 & Normal \\
$\begin{array}{c}\text { number) } \\
\text { Bells Test: omissions (left minus } \\
\text { right) } \\
\text { Bells Test: starting point }\end{array}$ & 2 & 2 & Borderline \\
& 5 & 5 & Impaired
\end{tabular}

Verbal episodic memory

$\begin{array}{llll}\text { CVLT }^{3} \text { total recall } & 22 & \mathrm{Z}=-3.4 & \text { Impaired } \\ \text { CVLT delayed recall } & 3 & \mathrm{Z}=-3.44 & \text { Impaired } \\ \text { CVLT cued delayed recall } & 6 & \mathrm{Z}=-2.6 & \text { Impaired }\end{array}$

Executive function/planning

Tower of London ${ }^{4}$

Not ended

Impaired

Executive function/flexibility

Color Trail Test Part $2^{5}$

Percentile $<1 \quad$ Impaired

Executive function/problem solving

Brixton Test ${ }^{6}$ (number of errors)

38

34

Impaired

Executive function/initiation

Letter fluency test ${ }^{7}$

3

5

Impaired

Category fluency test ${ }^{7}$

19

12

Normal

Non-verbal fluency test ${ }^{8}$ : productivity 8 (number of unique designs)

Percentile $<2 \quad$ Impaired

Non-verbal fluency test: repetitions

$42.85 \%$

Percentile $<2 \quad$ Impaired

Executive function/inhibition

Stroop ${ }^{9}$ interference time

162

117 (27)

Normal

Stroop errors

6

$0.36(0.93)$

Impaired

Behavioural Assessment of the Dysexecutive

$12 / 24$

Syndrome ${ }^{10}$ total profile score

Impaired

Alertness $^{11}$

Tonic arousal: reaction times (median)

Percentile 2 Impaired

Phasic arousal: reaction times (median) 338

Percentile 4 Impaired

Divided attention ${ }^{11}$

Auditory reaction times (median)

Percentile $<1 \quad$ Impaired

Visual reaction times (median)

Omissions

Errors

Percentile 4 Impaired

Percentile $1 \quad$ Impaired

Percentile 1 Impaired 
Running head: EXTERNAL DEVICE AND GOAL-DIRECTED BEHAVIOUR

Sustained attention ${ }^{11}$

Total reaction times (median)

Total $S D$

168

Total omissions

23

Total errors

42

86

Color Trail Test Part 1

Percentile $18 \quad$ Normal

Percentile 38 Normal

Percentile 3 Impaired

Percentile $<1 \quad$ Impaired

CVLT $=$ California Verbal Learning Test

*All tests are matched by age and by education. **Impaired = when R.Z.'s performance

is inferior to $M-1.65(S D)$, is below percentile 5 , or is below the borderline score.

${ }^{1}$ Hooper, 1958; ${ }^{2}$ Gauthier, Dehaut, \& Joanette, 1989; ${ }^{3}$ Poitrenaud, Deweer, Kalafat, \&

Van der Linden, 2007; ${ }^{4}$ Shallice, 1982; ${ }^{5}$ 'Elia, Satz, Uchiyama, \& White, 1996;

${ }^{6}$ Burgess \& Shallice, 1996; ${ }^{7}$ Thuillard \& Assal, 1989; ${ }^{8}$ Regard, Strauss, \& Knapp, 1982;

${ }^{9}$ GREFEX, 2001; ${ }^{10}$ Wilson, Alderman, Burgess, Emslie, \& Evans, 1996;

${ }^{11}$ Zimmermann \& Fimm, 2009. 
Running head: EXTERNAL DEVICE AND GOAL-DIRECTED BEHAVIOUR

Table 2. DEX scores before and after the intervention

\begin{tabular}{lcccccc}
\hline & \multicolumn{3}{c}{ Before } & & \multicolumn{3}{c}{ After } \\
DEX factors & R.Z. & Mother & Nurse & R.Z. & Mother & Nurse \\
\hline Initiate, sustain & 14 & 21 & 19 & 9 & 17 & 16 \\
Impulse control, & 6 & 4 & 7 & 5 & 3 & 3 \\
sequencing & 9 & 4 & 6 & 5 & 7 & 5 \\
Excitability & 4 & 8 & 3 & 3 & 9 & 8 \\
Regard for social standards & 33 & 37 & 35 & 22 & 36 & 32 \\
Total & & & & & & \\
\hline
\end{tabular}


Running head: EXTERNAL DEVICE AND GOAL-DIRECTED BEHAVIOUR

Figure Captions

Figure 1. Graphical representation of the intervention.

Figure 2. Graphical representation of data obtained from the first intervention programme regarding the percentage of R.Z.'s attendance at the workshops. The dashed lines represent trend (ordinary least squares).

Figure 3. Graphical representation of data obtained from the first intervention programme regarding the percentage of R.Z.'s attendance at the workshops. The dashed lines represent within-phase medians.

Figure 4. Graphical representation of data obtained from the second intervention programme regarding the percentage of R.Z.'s attendance at the workshops. The dashed lines represent trend (ordinary least squares).

Figure 5. Graphical representation of data obtained from the second intervention programme regarding the percentage of R.Z.'s attendance at the workshops. The dashed lines represent within-phase medians. 


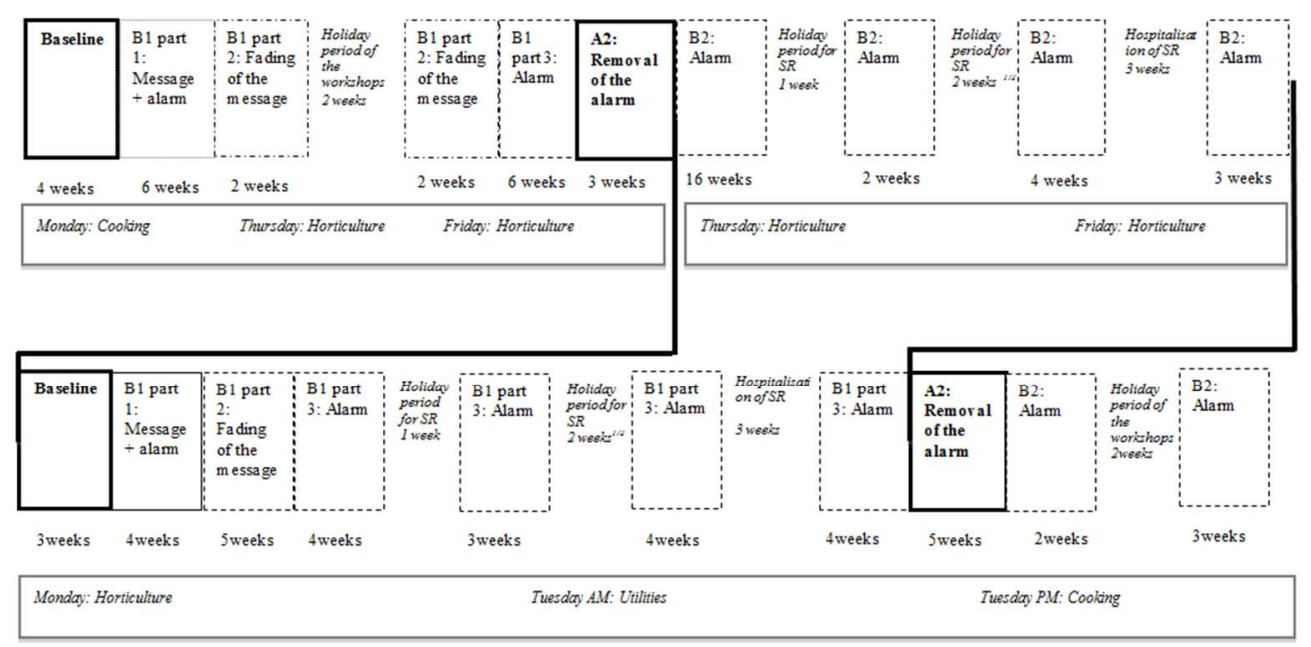

Figure 1. Graphical representation of the intervention. --INSERT FIGURE 1 HERE--

$262 \times 132 \mathrm{~mm}(96 \times 96 \mathrm{DPI})$ 


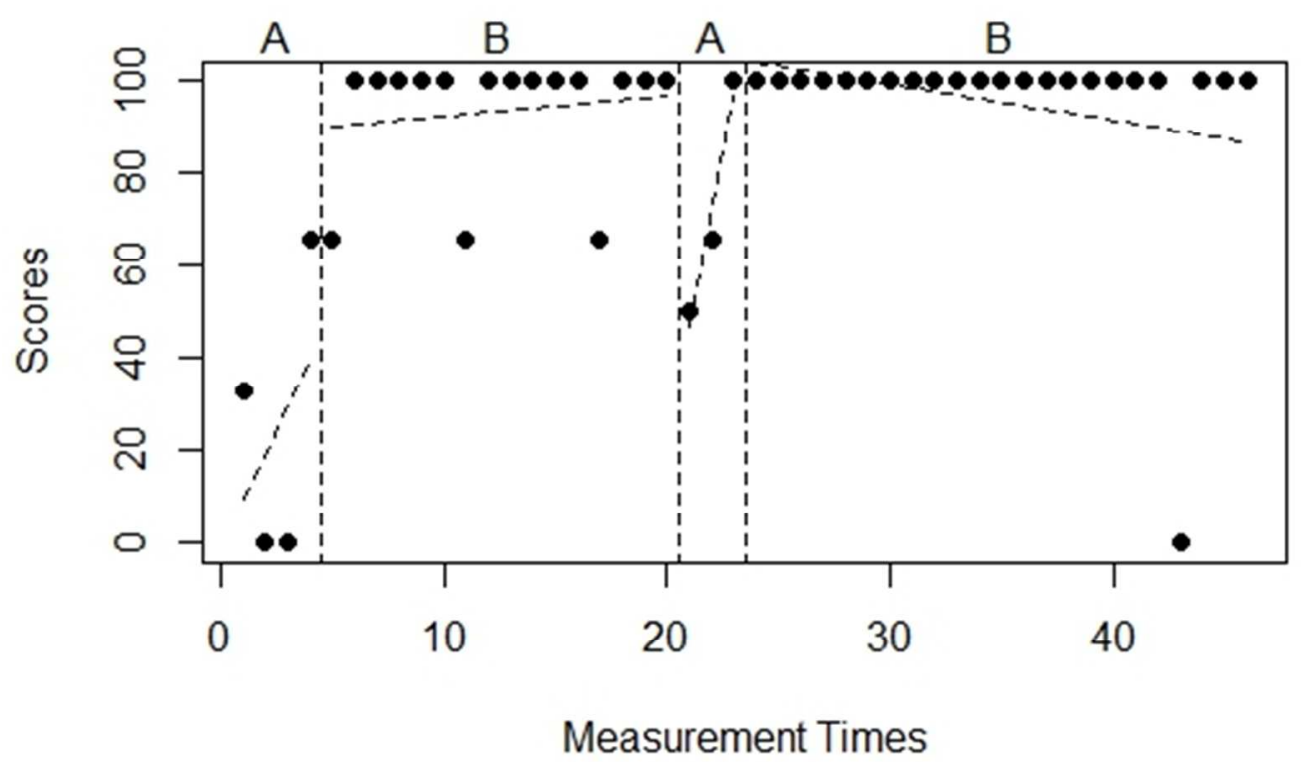

Figure 2. Graphical representation of the stage 1 data obtained regarding the percentage of S.R.'S attendance at the workshops. The dashed lines represent trend (ordinary least squares). --INSERT FIGURES 2 \& 3 HERE-$133 \times 78 \mathrm{~mm}(96 \times 96 \mathrm{DPI})$ 


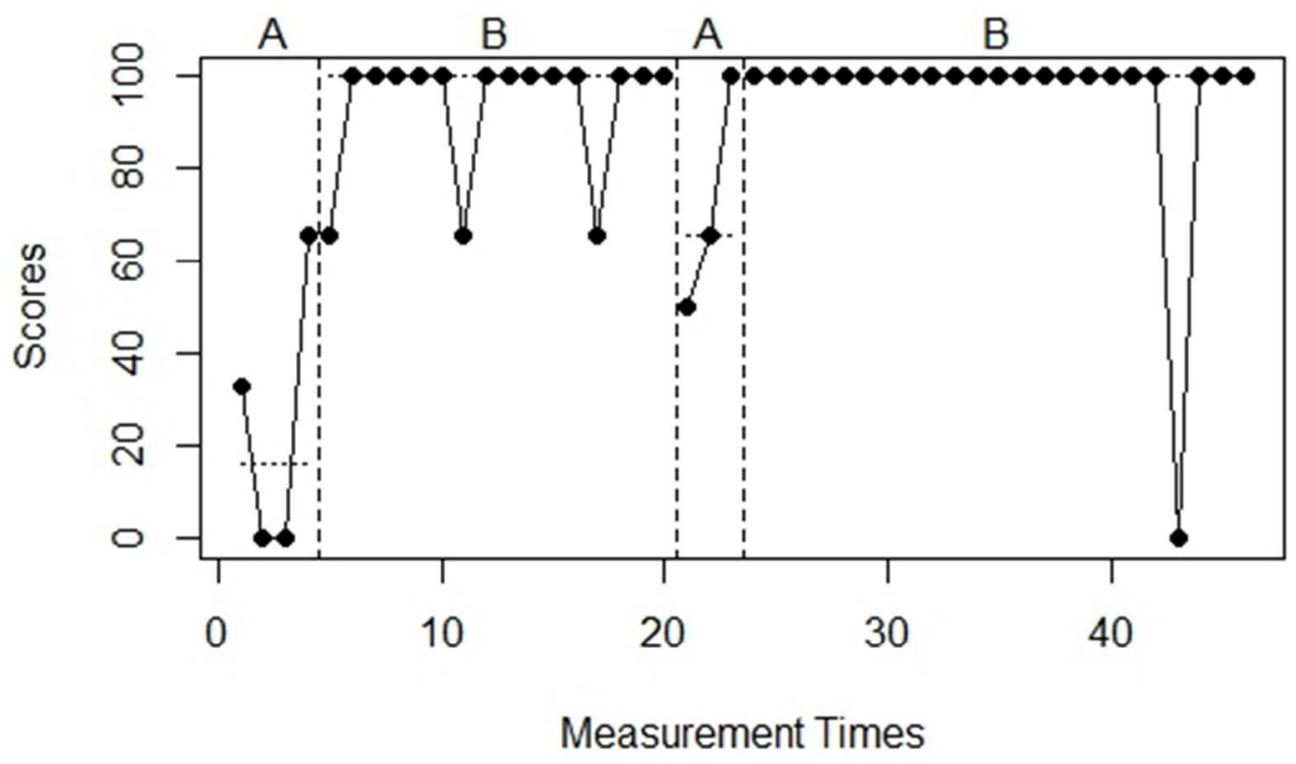

Figure 3. Graphical representation of the stage 1data obtained regarding the percentage of S.R.'s attendance at the workshops. The dashed lines represent within-phase medians.

--INSERT FIGURES 2 \& 3 HERE-$133 \times 78 \mathrm{~mm}(96 \times 96 \mathrm{DPI})$ 


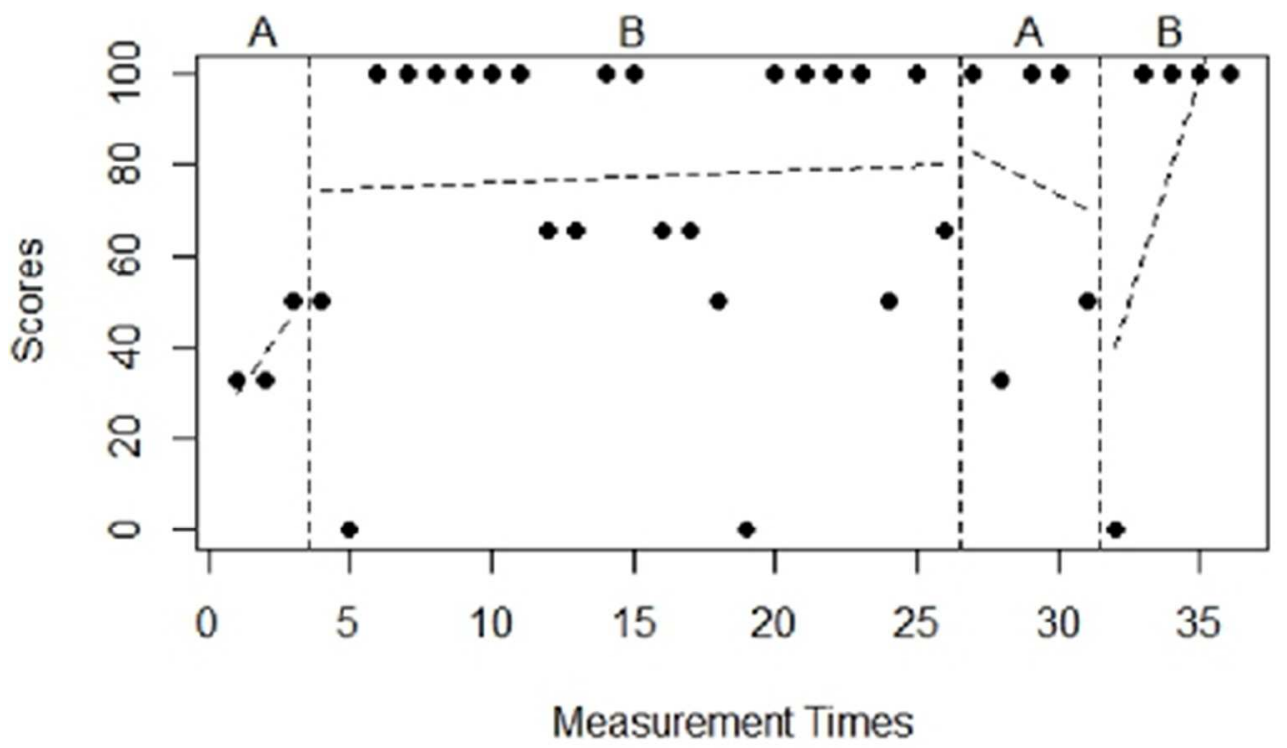

Figure 4. Graphical representation of the stage 2 data obtained regarding the percentage of S.R.'s attendance at the workshops. The dashed lines represent trend (ordinary least squares). --INSERT FIGURES 4 \& 5 HERE-$137 \times 81 \mathrm{~mm}(96 \times 96 \mathrm{DPI})$ 
Figure 5. Graphical representation of the stage 2 data obtained regarding the percentage of S.R.'S attendance at the workshops. The dashed lines represent within-phase medians.

--INSERT FIGURES 4 \& 5 HERE-$133 \times 80 \mathrm{~mm}(96 \times 96 \mathrm{DPI})$ 